\title{
A miniature all-optical photoacoustic imaging probe
}

\author{
Edward Z. Zhang* and Paul C. Beard \\ Department of Medical Physics and Bioengineering, University College London, \\ Gower Street, London WC1E 6BT, UK \\ http://www.medphys.ucl.ac.uk/research/mle/index.htm
}

\begin{abstract}
A miniature (250 $\mu \mathrm{m}$ outer diameter) photoacoustic probe for endoscopic applications has been developed. It comprises a single delivery optical fibre with a transparent Fabry Perot (FP) ultrasound sensor at its distal end. The fabrication of the sensor was achieved by depositing a thin film multilayer structure comprising a polymer spacer sandwiched between a pair of dichroic dielectric mirrors on to the tip of a single mode fiber. The probe was evaluated in terms of its acoustic bandwidth and sensitivity. Ultra high acoustic sensitivity has been achieved with a concave FP interferometer cavity design, which effectively suppresses the phase dispersion of multiple reflected beam within the cavity to achieve high finesse. The noise equivalent noise (NEP) achieved is 8 Pa over a $20 \mathrm{MHz}$ bandwidth. Backward mode operation of the probe is demonstrated by detecting photoacoustic signals in a variety of phantoms designed to simulate endoscopic applications. A side-viewing probe is also demonstrated illustrating an all-optical design for intravascular imaging applications.
\end{abstract}

Keywords: Photoacoustics, endoscopic imaging, intravascular imaging, Fabry Perot sensor, ultrasound, hydrophone, OCT

\section{INTRODUCTION}

There are a number of important clinical applications of photoacoustic imaging in which the target tissue can only be accessed by introducing an endoscopic probe percutaneously or through a natural orifice. Among these are the assessment of coronary artery disease, prostate cancer and gastrointestinal pathologies. The design of a photoacoustic probe for endoscopic applications poses several challenges. These include the question of how to integrate the delivery optical fibre with the ultrasound receiver without obscuring the excitation laser pulses, obtaining the necessary level of miniaturisation and achieving low unit cost for single use applications. To address these challenges a range of miniature all-optical photoacoustic (PA) probes which employ a transparent Fabry Perot ultrasound sensor at the tip of an optical fiber is envisaged. There are several advantages of this approach over conventional piezoelectric based photoacoustic probes. It offers a high level of miniaturisation, inexpensive batch fabrication and can readily be combined with other imaging modalities such as OCT and pulse-echo ultrasound. The concept also offers flexibility in its configuration allowing the implementation of forward and sideways looking probes and multielement imaging devices. To demonstrate the principle, a miniature ( $250 \mu \mathrm{m}$ outer diameter) single element forward-looking probe and a sideways looking imaging probe have been fabricated. This was achieved by forming a thin film multilayer structure comprising a polymer spacer sandwiched between a pair of dichroic dielectric mirrors on to the tip of a single mode fiber.

In our previous work on a fiber optic hydrophone [1 \& 2], a planar cavity structure was used as the FP sensor. The nominal diameter of the single mode optical fiber core which guides the FP sensor interrogation light is only around 10 $\mu \mathrm{m}$ [2]. The divergence of the interrogation light exiting the fiber core is significant when it enters the FP sensor element directly attached to the fiber distal end. The phase dispersion of the divergent, multiply reflected interrogation light within the FP cavity degrades the fringe visibility of the interferometer transfer function and thus also the phase sensitivity of the FP sensor. Consequently, the spacer thickness of our planar FP sensor based fiber optic probe is limited to $\sim 10 \mu \mathrm{m}$ and the noise equivalent pressure is $\sim 1 \mathrm{kPa}$. To increase the acoustic sensitivity of a fiber optic FP sensor probe, measures need to be taken to suppress the phase dispersion of the interrogation light resonating within the interferometer cavity in order to allow the use of a thicker FP cavity spacer without degrading the finesse of the

* ezzhang@medphys.ucl.ac.uk; phone +44 2076790281.

Photons Plus Ultrasound: Imaging and Sensing 2011, edited by Alexander A. Oraevsky, Lihong V. Wang, Proc. of SPIE Vol. 7899, 78991F • (c) 2011 SPIE. DOI: 10.1117/12.874883 
interferometer transfer function (ITF). Our approach in this work is to change the cavity geometry of the FP sensor attached directly to the fiber end, that is, to use a concave cavity structure.

In Section 2, the approach to implementing an all optical endoscopic imaging probe system is presented, along with configurations of forward and sideways looking probes. In Section 3, the fabrication of a concave FP cavity fiber optic sensor probe is described and the acoustic sensitivity of the probe is evaluated in terms of noise equivalent pressure. The backward mode operation of the probe system is demonstrated by delivering the excitation light along the optical fibre and through the FP sensor at the tip followed by detection of the PA signal induced from a black absorber phantom. Section 4 illustrates a sideways looking probe fabricated using a single mode optical fiber without introducing additional optical lens or prism.

\section{ARCHITECTURE OF THE ALL-OPTICAL ENDOSCOPIC IMAGING SYSTEM}

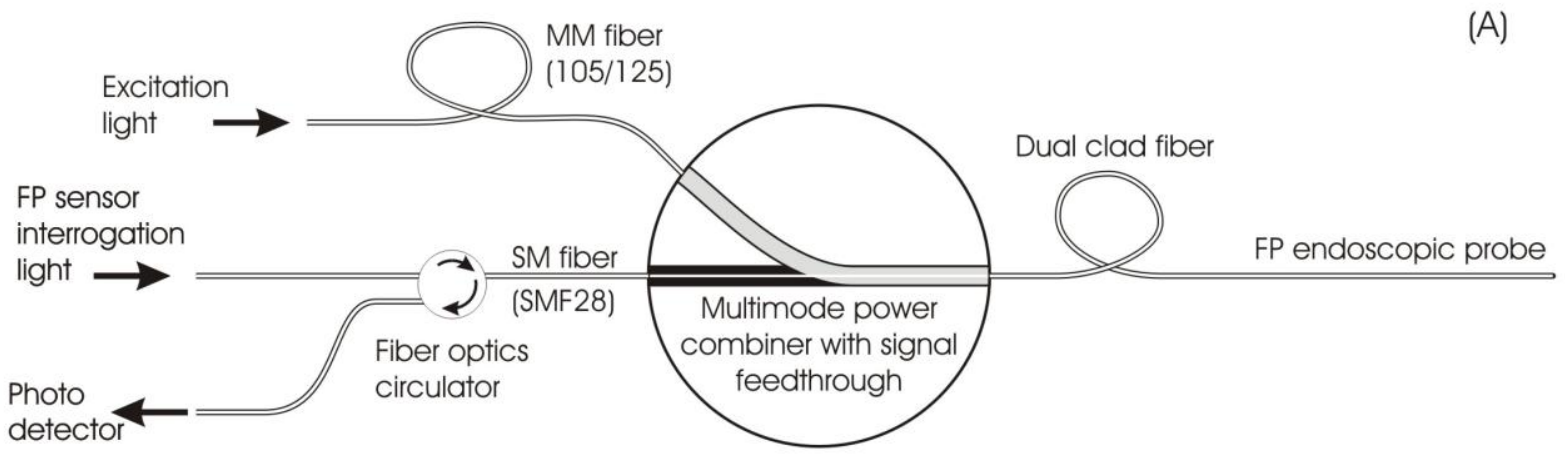

(B)

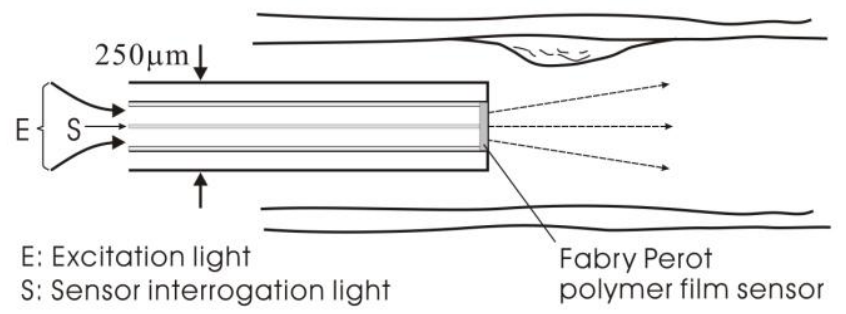

(C) Vessel wall

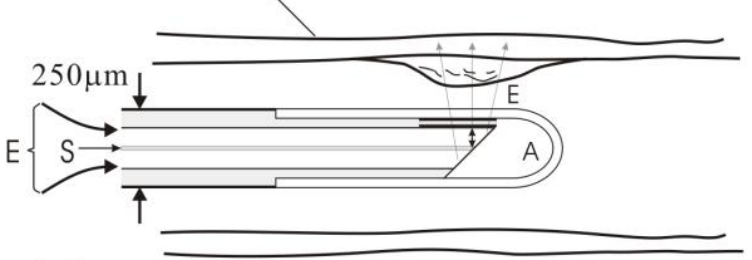

A: Encapsulated air

E: Excitation light

S: Sensor interrogation light

Figure 1: (A) Schematic of the all-optical endoscopic imaging system. (B) Forward looking probe. (C) Sideways looking scanning probe.

A schematic of the proposed all-optical endoscopic imaging system is depicted in Figure 1 (A). The configurations of two types of endoscopic probe, forward looking and sideways looking, are shown in Figure 1 (B) and (C), respectively. As shown in Figure 1 (B), the probe comprises a dual clad 1550nm single mode fiber and an acoustic sensing element, a FP polymer film sensor. The excitation and FP sensor interrogation lights are coupled into the dual clad probe fiber by a multimode pump combiner with $1550 \mathrm{~nm}$ single mode signal feed-through. The $1550 \mathrm{~nm}$ interrogation light delivered to and reflected from the FP sensor is guided within the single mode core of the fiber. The excitation light is delivered through the inner multimode cladding of the fiber so as to enable a sufficient amount of optical power to be transmitted. The FP sensor is designed to be transparent to excitation wavelengths ranging from $600 \mathrm{~nm}$ to $1200 \mathrm{~nm}$. A sideways looking probe can be made with a right angle prism attached to the fiber end and air encapsulated to maintain a total internal reflection (TIR) at the hypotenuse, as shown in Figure 1 (C). 


\section{CONCAVE FP SENSOR PROBE}

\subsection{Probe fabrication}

A concave cavity forward looking single element PA probe, as shown in Figure 2, was fabricated using a dual clad fiber (Fibercore SMM900). The cladding diameter of the fiber is $125 \mu \mathrm{m}$, the pump guide diameter is $100-104 \mu \mathrm{m}$ and the core is $10 \mu \mathrm{m}$ The structure of the FP sensor is illustrated in the same figure, of which the transmission spectrum of the concave dielectric mirror coatings is similar to that described in our previous work [3\&4]. The thickness of the polymer film spacer is $\sim 40 \mu \mathrm{m}$. That is four times thicker than the $10 \mu \mathrm{m} \_$spacer thickness limit for our planar cavity FP sensor probe [2]. The ITF of the concave FP sensor probe is depicted in Figure 3, and its finesse is almost 4 times that of its planar counterpart.

\subsection{Acoustic noise equivalent pressure of the probe}

The sensitivity of the concave FP sensor probe was evaluated in terms of noise equivalent pressure (NEP). The acoustic source used in the evaluation is a calibrated $3.5 \mathrm{MHz}, 25 \mathrm{~mm}$ diameter planar transducer with its output attenuated to $1.55 \mathrm{kPa}$. The probe sensor tip was placed $\sim 5 \mathrm{~mm}$ away from the transducer. The NEPs over a $20 \mathrm{MHz}$ bandwidth at various interrogation powers, were recorded and presented in Figure 4, showing a factor 75 of improvement compared to its planar counterpart.
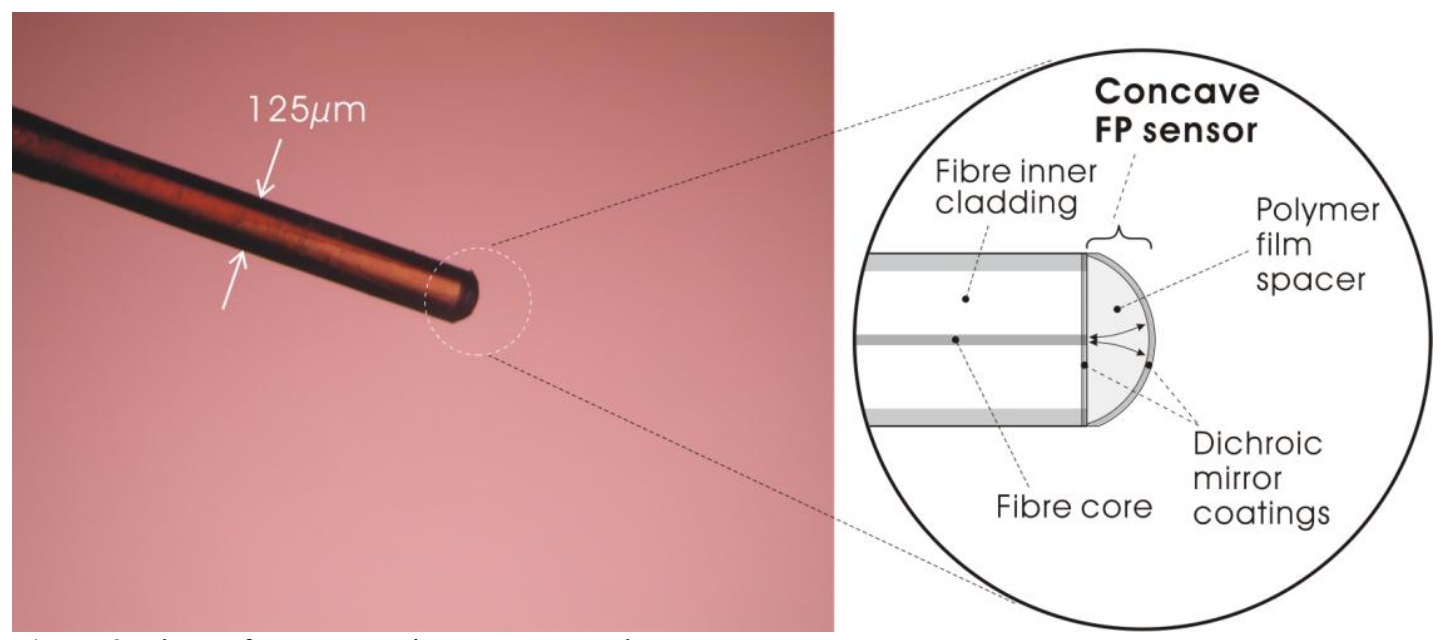

Figure 2: Photo of concave cavity FP sensor probe.

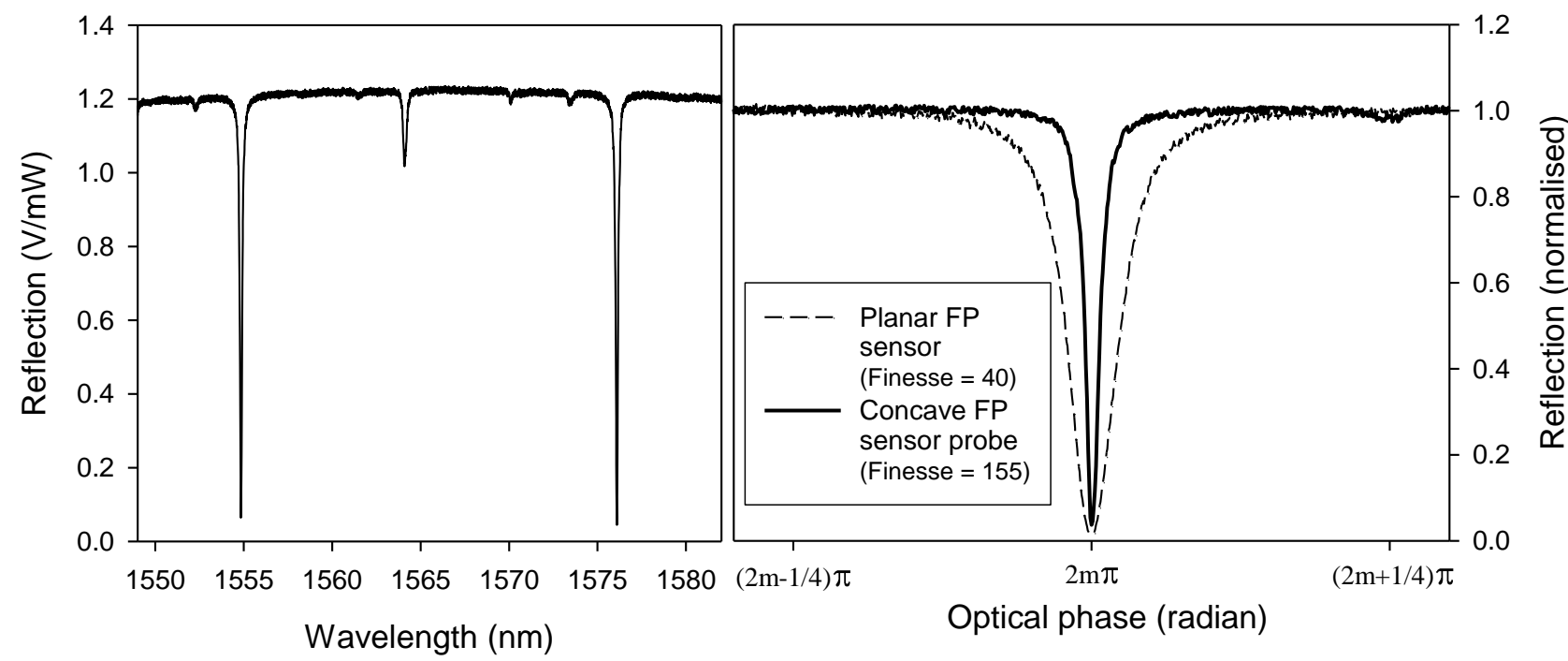

Figure 3: Interferometer transfer function (ITF) of the concave cavity FP sensor probe. 


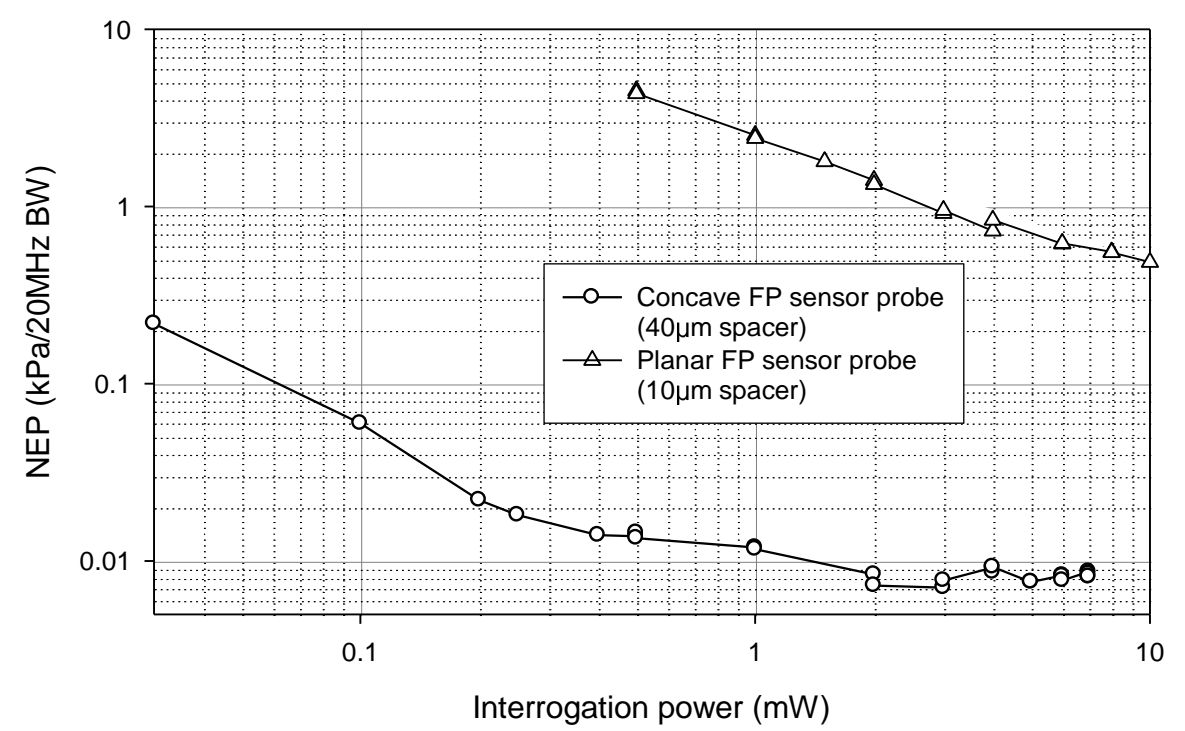

Figure 4: Noise equivalent pressure of the concave FP sensor probe vs optical interrogation power.

\subsection{Phantom study: PA signal detection in backward mode}

Backward mode PA signal detection with the concave FP sensor probe is demonstrated with the experimental setup depicted in Figure 5. The signal waveform recorded is also presented in the same figure. The excitation source is a 1064nm Q-switched Nd:YAG pulse laser. The excitation pulse energy exiting the probe and incident on the target is less than $20 \mu \mathrm{J}$. The PA signal induced from the phantom (black paint on PMMA substrate) is indicated in Figure 5. The signal fluctuation that occurs before the arrival of the PA signal from the phantom, as highlighted in the graph, is likely to be due to the impact of the excitation light pulse on the FP sensing element which appears to induce a transient temperature rise to due to absorption.
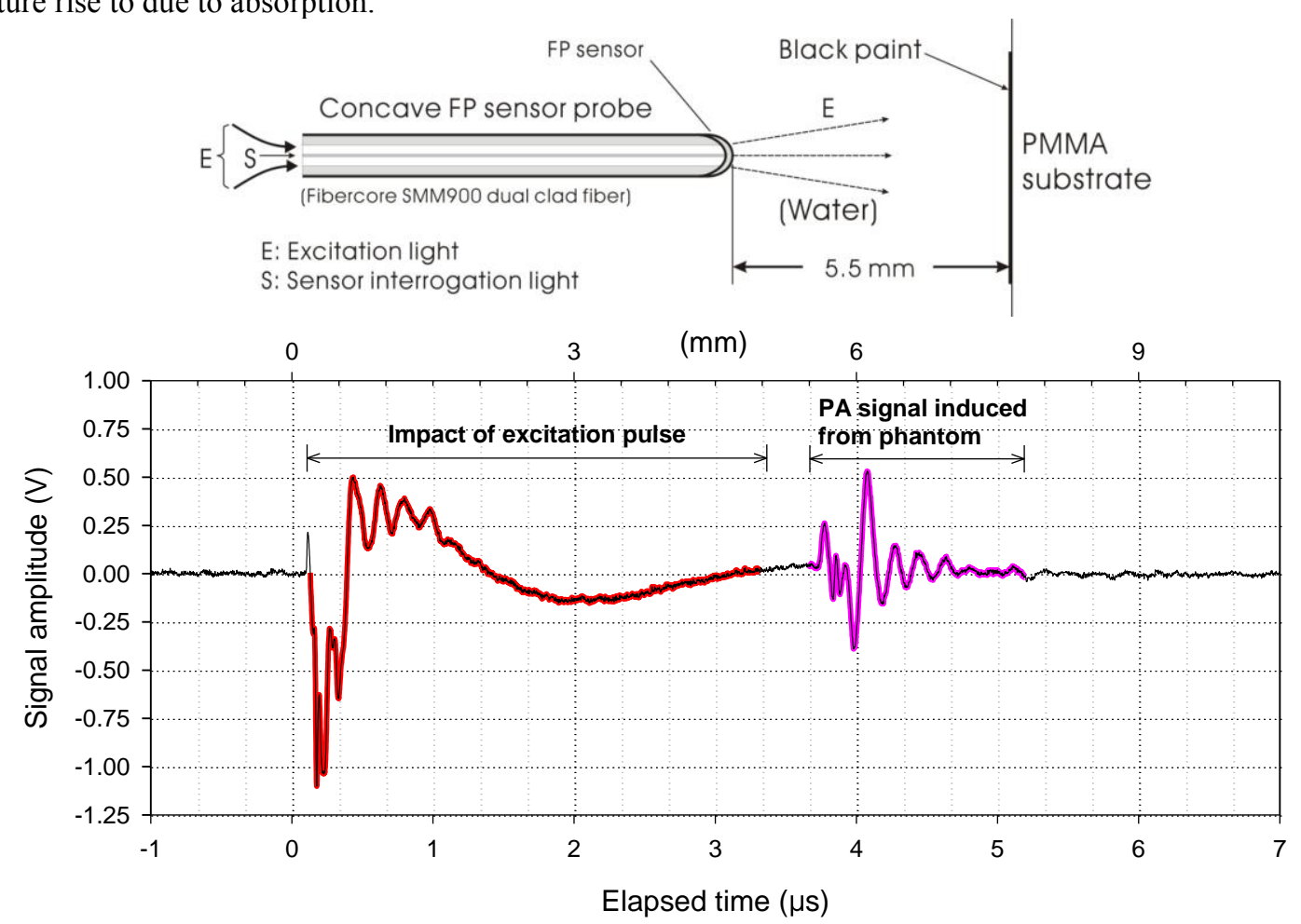

Figure 5: Forward looking, backward mode operation of the concave FP sensor probe and the PA signal waveform recorded. 


\section{SIDE-VIEWING SCANNING PROBE}

Figure 6 (A) illustrates a sideways looking probe fabricated using a single mode fiber. The fiber end was terminated with a $45^{\circ}$ angle by polishing. Air was encapsulated at the fiber end, so that the FP sensor interrogation light as well as the excitation light would be directed sideways by total internal reflection (TIP) at the glass/air interface, as shown in Figure 6 (A). The FP polymer film sensor was deposited on top of the fiber cladding, so that a concave cylindrical FP interferometer cavity was formed. As illustrated in Figure 6 (A), the interrogation light is well confined along the radial direction within the cavity. Along the axis of the fiber, the degree of the phase dispersion of this cylindrical cavity FP sensor probe is quite similar to that of a planar FP sensor probe, however its signal loss is expected to be higher than that of the latter. This is because that the beam directed sideways from the core is unguided and hence divergent, as shown in Figure 6 (A). Only a fraction of light reflected from FP sensor film $(\sim 62 \mu \mathrm{m}$ away from the fiber core) will be coupled back to the fiber. Experiments show that this probe is still able to collect $>30 \%$ of light reflected from the concave cylindrical cavity FP sensor film deposited on the surface of the fiber cladding.

\section{(A)}

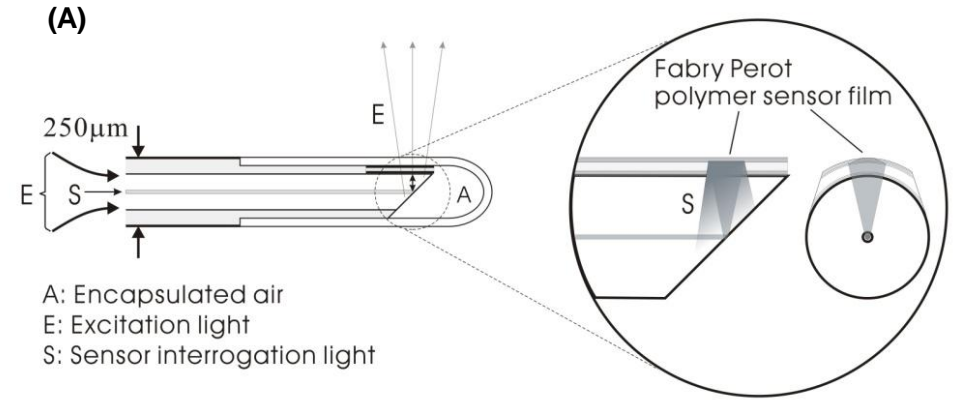

Figure 6: (A) A sideways looking probe with a concave cylindrical FP sensor cavity (B) PA signal produced by the absorption of $1064 \mathrm{~nm}$ pulsed laser light incident on a black absorber. This signal was detected with the probe operating in forward mode.

Figure 6 (B) depicts a PA signal waveform detected by this sideways looking probe with a cylindrical concave FP cavity. The PA signal was induced by the absorption of a 6.7ns, 1064nm Q-switched laser pulse in a planar black absorber (black paint on polymer substrate). The acoustic frequency response of the probe derived from the similar measurements is illustrated in Figure 7. Data obtained for spherical concave and planar cavity probes mentioned before are also depicted in the same graph.

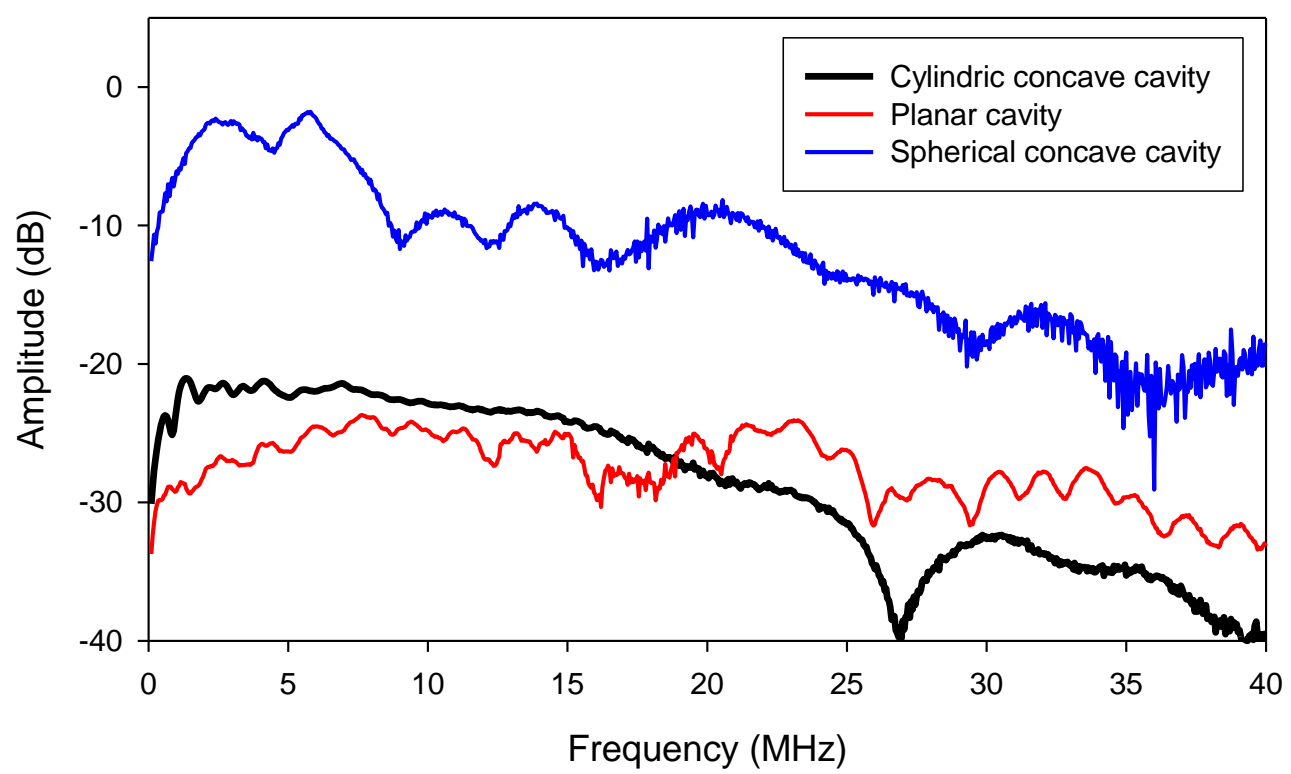

Figure 7: A sideways looking probe with a concave cylindrical FP sensor cavity. 


\section{CONCLUSIONS}

The transparent nature of the FP ultrasound sensor enables all optical photoacoustic imaging probes for endoscopic applications to be realised..This approach allows the minimum possible diameter of a photoacoustic probe to be achieved - ie one limited only by the diameter of the delivery optical fibre. This is demonstrated in this work by a single fiber optic endoscopic imaging probe of $250 \mu \mathrm{m}$ diameter. This work also illustrates that an ultra-sensitive FP sensor probe can be achieved with the use of a concave FP cavity geometry. It shows that with an all-optical design, the miniaturization of a PA probe can be achieved without compromising its acoustic sensitivity. The all-optical design of a

PA imaging probe also has the potential for multi-modal operation, and could be combined with OCT and other optical imaging and sensing methods.

\section{REFERENCES}

1 Beard, P. C., Perennes, F., Draguioti, E., and Mills, T. N., "An optical fibre photoacoustic-photothermal probe," Optics Letters 23(15), 1235-1237 (1998).

2 Morris, P. M., Hurrell, A., Shaw, A., Zhang, E., Beard, P. C., "A Fabry-Pérot fiber-optic ultrasonic hydrophone for the simultaneous measurement of temperature and acoustic pressure," Journal of the Acoustical Society of America, 125(6), 3611-3622 (2009).

3 Zhang, E., Laufer, J., Beard, P., "Backward-mode multiwavelength photoacoustic scanner using a planar Fabry-Perot polymer film ultrasound sensor for high-resolution three-dimensional imaging of biological tissues," Applied Optics 47, 561-577 (2008).

4 Zhang, E. Z., Laufer, J. G., Pedley, R. B., Beard, P. C., "In vivo high-resolution 3D photoacoustic imaging of superficial vascular anatomy," Physics in Medicine and Biology, 54, 1035-1046 (2009). 